\title{
Minimal Homozygous Endothelial Deletion of Eng with VEGF Stimulation Is Sufficient to Cause Cerebrovascular Dysplasia in the Adult Mouse
}

\author{
Eun-Jung Choi $^{\mathrm{a}}$ Espen J. Walker ${ }^{\mathrm{a}}$ Fanxia Shen ${ }^{\mathrm{a}} \quad$ S. Paul Oh ${ }^{\mathrm{d}}$ Helen M. Arthur \\ William L. Young ${ }^{\mathrm{a}-\mathrm{c}}$ Hua Su ${ }^{\mathrm{a}}$ \\ ${ }^{a}$ Department of Anesthesia and Perioperative Care, Center for Cerebrovascular Research, and Departments \\ of ${ }^{b}$ Neurological Surgery and ' Neurology, University of California, San Francisco, San Francisco, Calif., and \\ dDepartment of Physiology and Functional Genomics, Shands Cancer Center, University of Florida, Gainesville, Fla.,

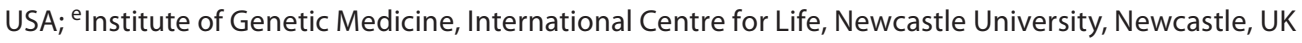

\section{Key Words}

Brain AVM - Animal model $\cdot$ Hereditary hemorrhagic telangiectasia $\cdot$ Angiogenesis $\cdot$ Mosaic gene deletion

\begin{abstract}
Background: Brain arteriovenous malformations (bAVMs) represent a high risk for hemorrhagic stroke, leading to significant neurological morbidity and mortality in young adults. The etiopathogenesis of bAVM remains unclear. Research progress has been hampered by the lack of animal models. Hereditary Hemorrhagic Telangiectasia (HHT) patients with haploinsufficiency of endoglin (ENG, HHT1) or activin receptor-like kinase 1 ( $A L K 1, \mathrm{HHT} 2$ ) have a higher incidence of BAVM than the general population. We previously induced cerebrovascular dysplasia in the adult mouse that resembles human bAVM through Alk1 deletion plus vascular endothelial growth factor (VEGF) stimulation. We hypothesized that Eng deletion plus VEGF stimulation would induce a similar degree of cerebrovascular dysplasia as the Alk1-deleted brain. Methods: Ad-Cre (an adenoviral vector expressing Cre recombinase) and AAV-VEGF (an adeno-associated viral vector expressing VEGF) were co-injected into the basal ganglia of 8- to 10-week-old Eng ${ }^{2 f / 2 f}$ (exons 5 and 6 flanked by loxP sequences), Alk $1^{2 f / 2 f}$ (exons $4-6$ flanked by loxP se-
\end{abstract}

quences) and wild-type (WT) mice. Vascular density, dysplasia index, and gene deletion efficiency were analyzed 8 weeks later. Results: AAV-VEGF induced a similar degree of angiogenesis in the brain with or without Alk1- or Eng-deletion. Abnormally patterned and dilated dysplastic vessels were found in the viral vector-injected region of $A l k 1^{2 f / 2 f}$ and Eng ${ }^{2 f / 2 f}$ brain sections, but not in WT. Alk $1^{2 f / 2 f}$ mice had about 1.8-fold higher dysplasia index than Eng ${ }^{2 f / 2 f}$ mice $(4.6 \pm 1.9$ vs. $2.5 \pm 1.1, p<0.05)$. However, after normalization of the dysplasia index with the gene deletion efficiency $\left(A / k 7^{2 f / 2 f}\right.$ : $16 \%$ and $E n g^{2 f / 2 f}: 1 \%$ ), we found that about 8 -fold higher dysplasia was induced per copy of Eng deletion (2.5) than that of Alk1 deletion (0.3). ENG-negative endothelial cells were detected in the Ad-Cre-treated brain of Eng ${ }^{2 f / 2 f}$ mice, suggesting homozygous deletion of Eng in the cells. VEGF induced more severe vascular dysplasia in the Ad-Cre-treated brain of $E_{n g}^{2 f / 2 f}$ mice than that of $E_{n g^{+/-}}$mice. Conclusions: (1) Deletion of Eng induces more severe cerebrovascular dysplasia per copy than that of Alk1 upon VEGF stimulation. (2) Homozygous deletion of Eng with angiogenic stimulation may be a promising strategy for development of a bAVM mouse model. (3) The endothelial cells that have homozygous causal gene deletion in AVM could be crucial for lesion development.

\section{KARGER}

Fax +4161306 1234

E-Mail karger@karger.ch

www.karger.com
(C) 2012 S. Karger AG, Basel

1015-9770/12/0336-0540\$38.00/0

Accessible online at:

www.karger.com/ced
Hua Su, MD

Department of Anesthesia and Perioperative Care

University of California, San Francisco, 1001 Potrero Avenue, Room 3C-38

San Francisco, CA 94110 (USA)

Tel. +1 415206 3162, E-Mail hua.su@ ucsf.edu 


\section{Introduction}

Brain arteriovenous malformations (bAVMs) cause spontaneous intracranial hemorrhage ( $\mathrm{ICH}$ ) leading to a high risk of stroke especially in children and young adults [1]. The pathogenesis and environmental risk factors for bAVMs remain elusive. The only available treatments for bAVM are removing the mass of abnormal vessels or occluding them by injecting embolic materials or by radiation. There is no specific medical therapy available to prevent the development or decrease the rate of bAVM rupture. Advances in bAVM research have been hindered by a lack of animal models for investigating disease mechanisms and testing new therapies. Although over 95\% of bAVMs are sporadic, the familial cases are primarily seen in patients with Hereditary Hemorrhagic Telangiectasia (HHT). HHT patients have a greatly increased prevalence of bAVM than the general population [2]. An autosomal dominant disorder, HHT is most commonly caused by functional haploinsufficiency in one of two genes: endoglin (ENG; HHT1) or activin receptor-like kinase 1 (ALK1; HHT2). Among subtypes, HHT1 patients tend to develop more bAVMs $(\sim 20 \%)$ than HHT2 patients ( 2\%) [3].

Endoglin and ALK1 are type III and type I transmembrane receptors, respectively, for the transforming growth factor- $\beta$ (TGF- $\beta$ ) superfamily [4]. Both of them are primarily expressed in endothelial cells (ECs) and highly induced in activated endothelium during angiogenesis. In general, ALK1 signaling regulates EC proliferation and migration, and its function is promoted by ENG [4]. Previously, we demonstrated that haploinsufficiency of either Eng $\left(E n g^{+/-}\right)$or Alkl $\left(A l k 1^{+/-}\right)$and an angiogenic insult are required for the development of cerebrovascular abnormalities in adult mice [5]. In addition, there were more dysplastic vessels formed in the brain of $\mathrm{Eng}^{+/-}$than $A l k 1^{+/-}$mice in response to vascular endothelial growth factor (VEGF) stimulation [5], which replicated the relative penetrance of bAVM observed in HHT patients. However, abnormal vessel formation in the brain of $\mathrm{Eng}^{+/-}$and $\mathrm{Alk1}^{+/-}$mice was at the capillary level, which recapitulated perinidal dysmorphic and dilated capillaries $[6,7]$, but not abnormally tangled large vessels seen in human bAVM nidus.

To accomplish homozygous Alk1-deletion in the adult mouse brain, we utilized the Cre/loxP system. This system includes a genetically modified mouse that has the targeted gene or a part of the target gene flanked by two loxP sequences. Cre recombinase can be introduced by transgenic techniques (establishment of a Cre driver transgenic mouse line or direct administration of Creexpressing vectors) or Cre protein administration. Cre mediates recombination of the two loxP sites, which results in the deletion of the sequence flanked by the loxP (online suppl. fig. 1; for all online suppl. material, see www.karger.com/doi/10.1159/000337762). In Alk1 $1^{2 f / 2 f}$ mice, exons 4-6 on both alleles are flanked by loxP sequences. By injecting Ad-Cre (an adenoviral vector expressing Cre) and AAV-VEGF into the basal ganglia, we deleted exons 4-6 of the Alk1 gene in that region (online suppl. fig. 2) and induced focal angiogenesis, resulting in a cerebrovascular phenotype that recapitulated many key aspects of human bAVM [8], including irregularly dilated vessel morphology, arteriovenous shunting, and inflammatory cell infiltration. Based on the observation that HHT1 patients have a higher incidence of bAVM than HHT2 patients [2] and our data that $\mathrm{Eng}^{+/-}$mice develop more dysplastic vessels in their brain than $A l k 1^{+/-}$mice upon VEGF stimulation [5], in this study, we tested the hypothesis that co-injection of Ad-Cre and AAV-VEGF into the brain of $E n g^{2 f / 2 f}$ mice induces more severe cerebrovascular dysplasia than $A l k 1^{2 f / 2 f}$ mice.

\section{Methods}

Animals

Experimental procedures for using laboratory animals were approved by the Institutional Animal Care and Use Committee (IACUC) of the University of California, San Francisco (UCSF). Adult (8- to 10-week-old) Alk1 $1^{2 f / 2 f}$ mice (exons 4-6 flanked by loxP sites) [9] and $E n g^{2 f / 2 f}$ (exons 5 and 6 flanked by loxP sites) [10] mice were used. Adult C57BL/6 wild-type (WT; Jackson Laboratory, Bar Harbor, Me., USA) mice were used as control. ROSA26 reporter (R26R; Jackson Laboratory) mice were used to test the deletion efficiency of the floxed allele (2f) by Ad-Cre stereotactic injection (online suppl. fig. 3).

\section{Viral Vector Gene Transfer}

Adenoviral vectors with cytomegalovirus (CMV) promoter driving Cre recombinase (Ad-Cre) or green fluorescent protein (Ad-GFP) expression were purchased from Vector Biolabs (Philadelphia, Pa., USA). Adeno-associated viral vectors with CMV promoter driving VEGF (AAV-VEGF) or $\beta$-galactosidase (AAVlacZ) expression were prepared as described previously $[11,12]$. Adenoviral $\left(2 \times 10^{7}\right.$ plaque forming unit $)$ and AAV $\left(2 \times 10^{9}\right.$ genome copies) vectors were co-injected stereotactically into the basal ganglia of mice in different combinations as described previously [8].

Vascular Casting with Microfil, in vivo Vascular Labeling with Lectin, Vascular Density, and Dysplasia Index Quantification

Procedures for vascular casting with microfil (Flow Tech Inc., Carver, Mass., USA) and in vivo vascular labeling with lectin (Vector Laboratories, Burlingame, Calif., USA) were described 
Table 1. Primers used for $q P C R$

\begin{tabular}{lll}
\hline Gene & Forward primer & Reverse primer \\
\hline Alk1 & CCTGGACAGCGACTGTACTAC & GCCCCATTGCTCTCCTCAAAC \\
Eng & GACGCCATTCTCATCCTGC & CCACGCCTTTGTCCTTGC \\
$M m p-9$ & GTGGGACCATCATAACATCACA & CTCGCGGCAAGTCTTCAGAGTA \\
\hline
\end{tabular}

previously $[8,13]$. Two coronal sections of the lectin-perfused brain per mouse, $0.5 \mathrm{~mm}$ rostral and $0.5 \mathrm{~mm}$ caudal to the injection site, were chosen and stained with an antibody against $\alpha$ smooth muscle actin ( $\alpha$-SMA; 1:1,000; Sigma-Aldrich, St. Louis, Mo., USA). Three areas (right and left of, and underneath the injection site) of each section were captured under $\times 20$ microscopic objective lens. Capillaries (lectin positive and $\alpha$-SMA negative) in each picture were counted separately using NIH Image 1.63 software by three blinded investigators $[5,8]$. Vascular density was calculated as the mean of capillaries obtained from 6 images per animal. A vessel was considered dysplastic when it had a diameter $>15 \mu \mathrm{m}$ and was $\alpha$-SMA negative [14]. In general, the diameter of a normal capillary is $3-8 \mu \mathrm{m}$ [15]. Dysplasia index was defined as the number of capillaries $>15 \mu \mathrm{m}$ per 200 capillaries.

\section{Immunohistochemistry}

Sections of the lectin-perfused brain were stained with primary antibodies against $\beta$-galactosidase (1:1,000, Abcam Inc., Cambridge, Mass., USA) or ENG (1:50; BD Pharmingen, Franklin Lakes, N.J., USA). Expression of $\beta$-galactosidase was subsequently detected by a fluorescent secondary antibody (Alexa Fluor 594 anti-rat IgG; Invitrogen, Carlsbad, Calif., USA). ENG-positive staining was visualized using a biotin-conjugated secondary antibody (anti-rat IgG; 1:500; Vector Laboratories) and the standard ABC method (Vector immunodetection kit; Vector Laboratories).

\section{Quantitative Real-Time PCR}

Genomic DNA was isolated from brain tissue around the virus injection sites and tails using the QIAamp DNA Micro Kit (Qiagen). Quantitative real-time PCR (qPCR) was performed using the QuantiTect SYBR Green PCR Kit (Qiagen) and PCR cycler (Mx3000P QPCR System, Agilent Technologies), following the manufacturer's protocol. The primers used for genomic DNA qPCR are summarized in table 1 .

\section{Statistical Analysis}

Data are presented as mean \pm SD. One-way analysis of variance (ANOVA) was used to determine a statistical significance among groups, followed by pairwise multiple comparisons using the post-hoc Tukey test. A p value $<0.05$ was considered statistically significant.

\section{Results}

Malformed vessels were observed in both $A l k 1^{2 f / 2 f}$ and $E n g^{2 f / 2 f}$ adult mouse brain at the Ad-Cre/AAV-VEGF injection site, while normal angiogenesis was observed in the WT brain (fig. 1). Histological analysis of lectin-perfused brain sections showed that WT mice treated with AAV-VEGF had higher mean vascular density than mice treated with AAV-lacZ $(202 \pm 43$ vs. $111 \pm 15, \mathrm{p}<0.05$; fig. 2a). VEGF induced a similar degree of angiogenesis in the experimental mice (WT: $180 \pm 43, A l k 1^{2 f / 2 f}: 219 \pm$ 49, and $E n g^{2 f / 2 f}: 195 \pm 59, \mathrm{p}=0.2$; fig. 2b). The dysplasia index was significantly higher in the brain of $A l k 1^{2 f / 2 f}$ and $E n g^{2 f / 2 f}$ mice injected with Ad-Cre/AAV-VEGF as compared to similarly treated WT mice $\left(A l k 1^{2 f / 2 f}: 4.6 \pm 1.9\right.$ and $E n g^{2 f / 2 f}: 2.5 \pm 1.1$ vs. WT: $0.5 \pm 0.4$, p $<0.05$; fig. 2 c). Injection of Ad-GFP (a control viral vector for Ad-Cre) with AAV-VEGF to Alk $1^{2 f / 2 f}$ or $E n g^{2 f / 2 f}$ mice led to normal angiogenesis (data not shown). Injection of AAV-lacZ (a control viral vector for AAV-VEGF) with Ad-Cre had no effect on the vascular density and structure (data not shown).

Interestingly, injection of Ad-Cre and AAV-VEGF induced more cerebrovascular dysplasia in $A l k 1^{2 f / 2 f}$ than Eng $^{2 f / 2 f}$ mice ( $4.6 \pm 1.9$ vs. $2.5 \pm 1.1, \mathrm{p}<0.05$; fig. $2 \mathrm{c}$ ), which contradicted our hypothesis. To investigate why $A l k 1^{2 f / 2 f}$ mice had a more severe dysplastic phenotype than $E n g^{2 f / 2 f}$ mice, we analyzed the gene deletion efficiency mediated by Ad-Cre. The effectiveness of Ad-Cre-mediated floxed allele (2f) deletion has been tested in ROSA26 reporter (R26R) mice [8]. In R26R mice, a transgene containing the loxP-flanked STOP cassette (stopper) introduced between the promoter and lacZ reporter gene is inserted into the ROSA 26 locus. In the presence of Cre, the stopper is deleted by recombination, followed by activation of lacZ expression (online suppl. fig. 3). Further analysis showed that Ad-Cre-mediated homozygous gene deletion (lacZ gene expression) demonstrated a mosaic pattern in some of the ECs (fig. 3a). ENG-negative ECs were detected in the angiogenic foci of the Ad-Cre/ AAV-VEGF-injected $E n g^{2 f / 2 f}$ brain (fig. 3b), suggesting that Ad-Cre-mediated homozygous Eng deletion in some of the ECs.

To quantify the gene deletion efficiency, we performed qPCR. We first examined the level of Alk1- and Engfloxed alleles that contain two loxP sites (2f) in genomic tail DNA. Matrix metalloproteinase- $9(\mathrm{Mmp}-9)$ gene was 


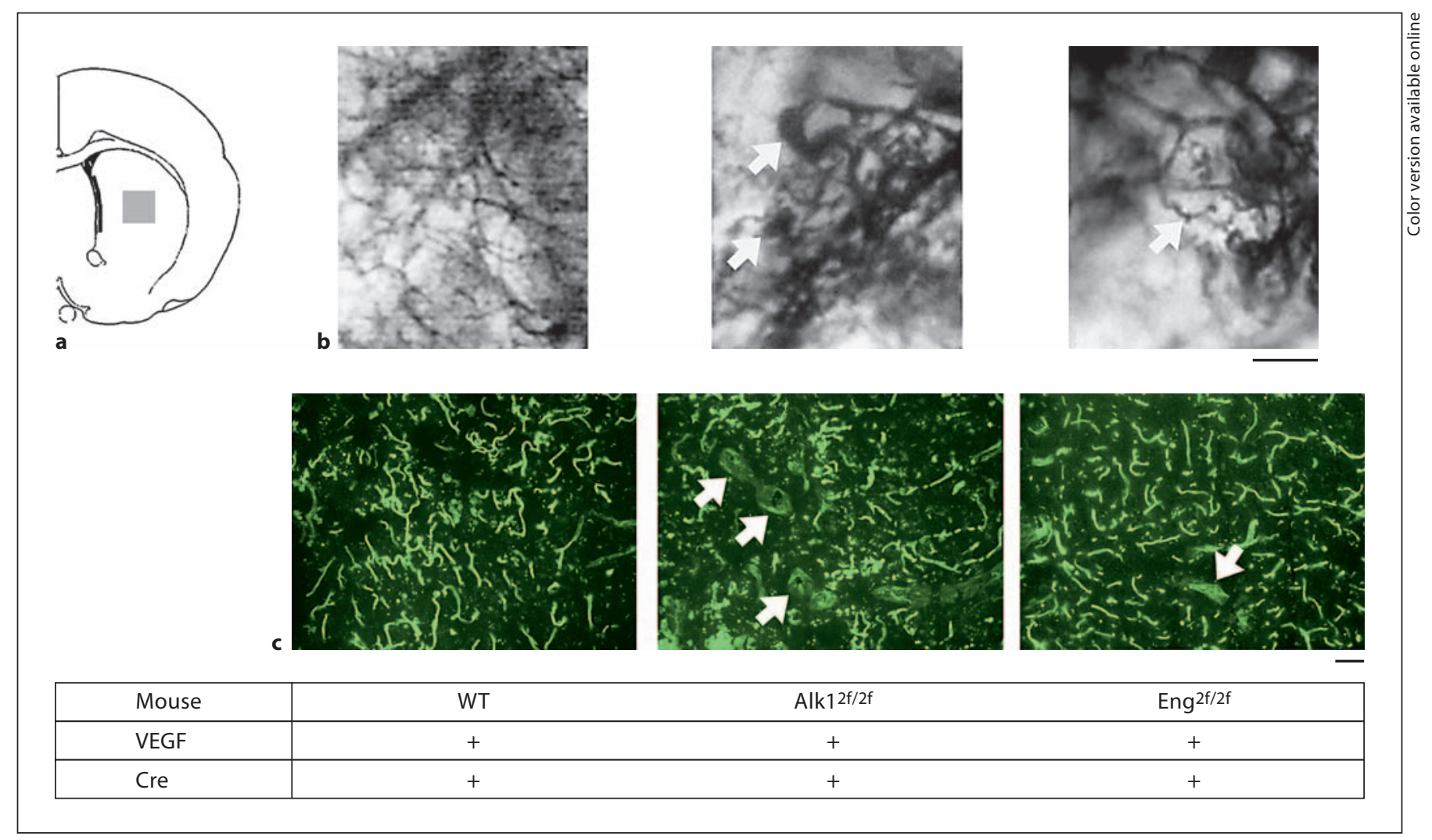

Fig. 1. Dysplastic cerebral vasculature was detected in the AdCre and AAV-VEGF-injected brain of $A l k 1^{2 f / 2 f}$ and $E n g^{2 f / 2 f}$ mice. a Diagram indicates the viral vector injection site (gray square). b Representative images of the microfil-perfused brain. Injection of Ad-Cre with AAV-VEGF to the WT brain caused focal normal angiogenesis, while it induced localized dysplastic vessel forma- tion in the $A l k 1^{2 f / 2 f}$ and $E n g^{2 f / 2 f}$ brain (arrows). c Representative images of lectin-perfused brain sections. Dysplastic vessels were observed in the Ad-Cre/AAV-VEGF-injected Alk $1^{2 f / 2 f}$ and $E n g^{2 f / 2 f}$ brain (arrows), but not in the similarly treated WT brain. Scale bars are $100 \mu \mathrm{m}(\mathbf{b})$ and $50 \mu \mathrm{m}(\mathbf{c})$.

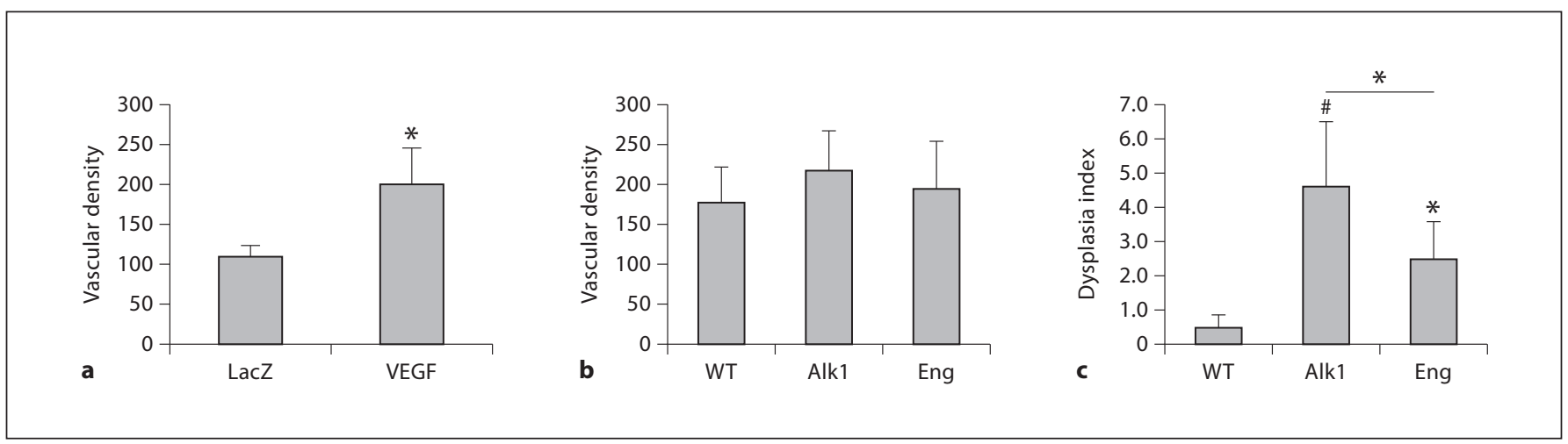

Fig. 2. Injection of Ad-Cre and AAV-VEGF increased cerebrovascular density and induced cerebrovascular dysplasia in $A l k 1^{2 f / 2 f}$ and $E n g^{2 f / 2 f}$ mice. a Quantification of vascular density in the brain of WT mice injected with AAV-VEGF or AAV-lacZ. b Bar graph shows the mean vascular densities in the brain of WT, Alk $1^{2 f / 2 f}$, and $E n g^{2 f / 2 f}$ mice injected with Ad-Cre and AAV-VEGF. c Bar graph shows dysplasia index. LacZ: AAV-lacZ; VEGF: AAVVEGF; WT, Alk1, and Eng: WT, $A l k 1^{2 \mathrm{f} / 2 \mathrm{f}}$, and $E n g^{2 \mathrm{f} / 2 \mathrm{f}}$ mice treated with Ad-Cre and AAV-VEGF. ${ }^{*} \mathrm{p}<0.05$ and ${ }^{\#} \mathrm{p}<0.001 . \mathrm{n}=6$ per group. 

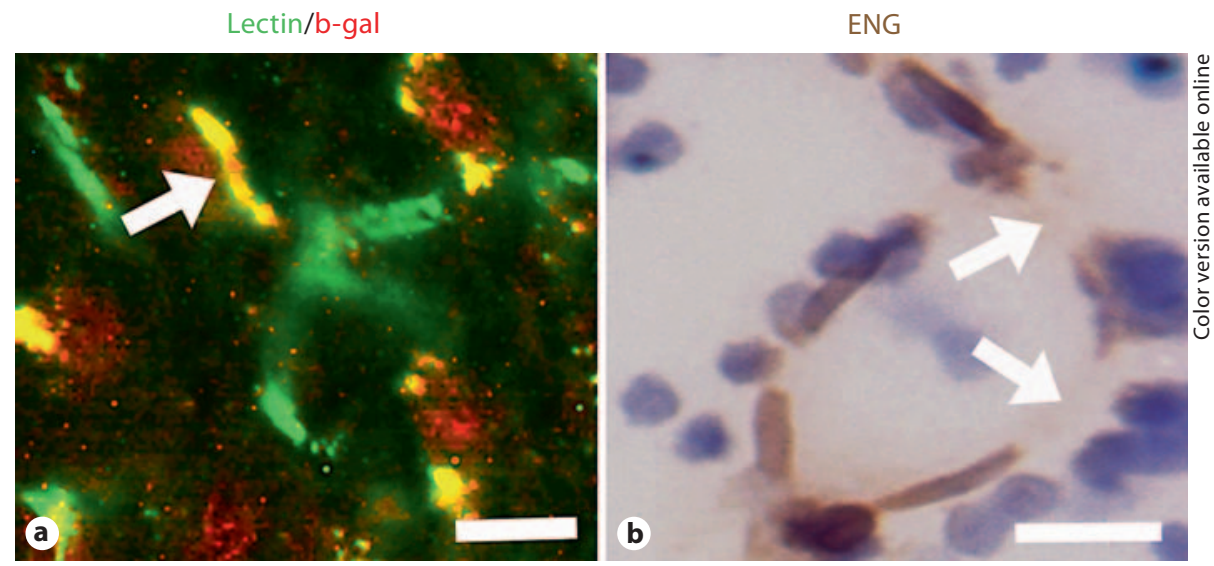

Fig. 3. Injection of Ad-Cre-mediated target gene deletion in the viral vector-injected region of the adult mouse brain. a Representative image of Ad-Cre-treated R26R mouse brain sections. The vessels were perfused with lectin, and lacZ expression was detected by immunostaining. Deletion of the floxed allele was observed in ECs (arrow). b Representative image of Ad-Cre-treated
Eng ${ }^{2 f / 2 f}$ mouse brain sections shows enlarged dysplastic vessels. ENG expression was detected by immunostaining. Hematoxylin was used for counterstaining. A mosaic pattern of ENG expression was detected in the ECs lining this vessel; a majority of ECs expressed ENG, two ECs were ENG negative (arrow). Scale bars are $20 \mu \mathrm{m}$.
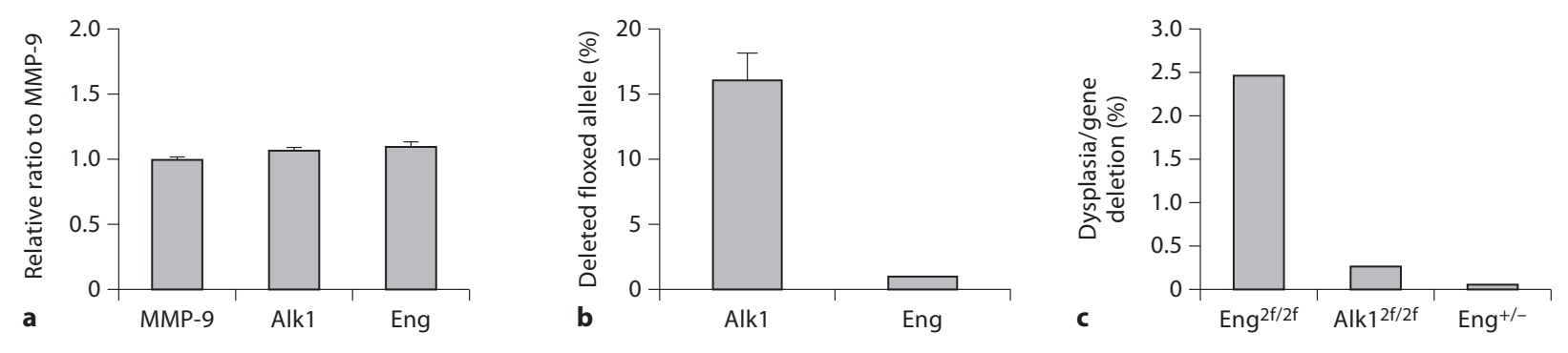

Fig.4. Ad-Cre mediated more effective deletion of the Alk1-floxed allele than Eng-floxed allele in the adult mouse brain. a Bar graph shows the amounts of Alk1- and Eng-floxed alleles and Mmp-9 in tail genomic DNA. The mean value of $M m p-9$ was set as a value of 1. Values of Alk1- and Eng-floxed alleles were presented as the ratios to Mmp-9. b Bar graph shows percentage of deleted Alk1and Eng-floxed alleles in the Ad-Cre-injected brain of $A l k^{2 f / 2 f}$ and $E n g^{2 f / 2 f}$ mice. c Bar graph shows dysplasia per percentage of gene deletion. $n=3$ per group in $\mathbf{a}$ and $\mathbf{b}$, and $n=6$ per group in $\mathbf{c}$. used as an internal quantitative control. When we compared Alk1- and Eng-floxed alleles to $M m p$-9, the ratio was 1:1 in both instances, confirming that the qPCR condition we used could amplify both floxed alleles and an internal positive control gene in an unbiased way (fig. 4a). Next, the copies of Alk1- or Eng-floxed alleles in the AdCre-treated brain sections were analyzed. Genomic DNA was isolated from brain tissue containing the angiogenic foci where the cells were infected with both Ad-Cre and AAV-VEGF. Ad-Cre and AAV-VEGF-injected Alk $1^{2 f / 2 f}$ and $E n g^{2 f / 2 f}$ mice had similar vessel densities in the viral vector injection sites, suggesting that Ad- and AAV-mediated gene transduction and expression were equivalent in these two groups of mice. The copy numbers of Alk1and Eng-floxed $2 \mathrm{f}$ alleles were normalized to the copy number of $M m p-9$. The gene deletion was determined by comparing the copies of the floxed allele in the brain to that in the tail. About $16 \%$ of the Alk1-floxed allele was deleted in the Ad-Cre-injected brain of $A l k 1^{2 f / 2 f}$ mice, while only 1\% of the Eng-floxed allele was deleted in the brain of similarly treated $E n g^{2 f / 2 f}$ mice (fig. 4b). Thus, more severe dysplasia seen in $A l k 1^{2 f / 2 f}$ mice compared to 
$E n g^{2 f / 2 f}$ mice appears to be due to more effective gene deletion.

To analyze whether similar dysplasia would have developed in the brain of $E n g^{2 f / 2 f}$ and $A l k 1^{2 f / 2 f}$ mice, if the gene deletion efficiency was the same, we divided the mean dysplasia index of these mice by the mean deletion efficiency of each gene. We found that deletion of Eng resulted in more dysplastic vessels per gene copy than that of $A l k 1$ in the adult mouse brain (Eng $g^{2 f / 2 f}: 2.5 \mathrm{vs}$. Alk $1^{2 f / 2 f}$ : 0.3; fig. 4c). To test if homozygous deletion of Eng is a more potent dysplasia inducer than heterozygous deletion, the value of dysplasia index per deleted gene copy in $E n g^{2 f / 2 f}$ mice was further compared with that observed in our previously published study using $\mathrm{Eng}^{+/-}$mice that had $50 \%$ gene deletion [5]. Eng ${ }^{2 f / 2 f}$ mice treated with Ad-Cre/ AAV-VEGF had a higher dysplasia index/deletion of gene copy than $\mathrm{Eng}^{+/-}$mice treated with AAV-VEGF [5] $\left(E n g^{2 f / 2 f}: 2.5\right.$ vs. $E n g^{+/-}: 0.06$; fig. $\left.4 c\right)$. $E n g^{2 f / 2 f}$ mice also had more severe vascular dysplasia (larger dysplastic vessels at a macroscopic level; fig. 1) than $E n g^{+/-}$mice in which the dysplasia was at the capillary level [5] (fig. 4c), suggesting that homozygous deletion of Eng is necessary in the induction of severe cerebrovascular dysplasia.

\section{Discussion}

In this study, we compared the effectiveness of AdCre and AAV-VEGF in the induction of cerebrovascular malformation in adult $A l k 1^{2 f / 2 f}$ and $E n g^{2 f / 2 f}$ mice. We found that (1) Ad-Cre-mediated gene deletion was less effective in the brain of $E n g^{2 f / 2 f}$ mice compared to $A l k 1^{2 f / 2 f}$ mice, and (2) more dysplastic vessels were induced per copy of Eng deletion than that of Alk1 deletion after VEGF stimulation. In addition, VEGF caused more severe dysplasia (macroscopic level) in the Ad-Cre-injected $E n g^{2 f / 2 f}$ brain than in the Eng ${ }^{+/-}$brain (capillary level), suggesting that homozygous deletion of Eng is more potent in the induction of vascular dysplasia and may be necessary for the development of a fully-formed AVM. Therefore, these observations are consistent with the notion that loss of heterozygosity of Eng (Eng-null) due to somatic mutations in the normal allele in a subset of ECs - perhaps quite small - could be the trigger for AVM development in HHT.

The R26R transgenic mice have been used to assess the efficiency of Cre-mediated conditional gene deletion in various organs and cell types [16-19]. We previously demonstrated that more than $50 \%$ of ECs, neurons, and astrocytes in the Ad-Cre-injected basal ganglia of R26R mice showed lacZ expression by immunohistochemical analysis, indicating that Ad-Cre-mediated gene deletion efficiency in the brain of R26R mice was $>50 \%$ [8]. Here, using qPCR analysis, we found that there was much less gene deletion in the brain of $A l k 1^{2 f / 2 f}$ and $E n g^{2 f / 2 f}$ mice with the same Ad-Cre viral vector, virus dose, and injection method. We showed a similar degree of angiogenesis in the brain of $A l k 1^{2 f / 2 f}$ and $E n g^{2 f / 2 f}$ mice induced by coinjection of Ad-Cre and AAV-VEGF, indicating the same degree of viral transduction. Further, Cre-mediated gene deletion was evidenced by reporter gene expression and reduced expression of the target gene in the angiogenic foci (fig. 3). However, we observed different gene deletion efficiency in these mice (Alk1: 16\% vs. Eng: 1\%). Thus, the efficiency of Cre-mediated gene deletion varies in targeted genes, suggesting that R26R mice may not be a reliable model to assess effectiveness of Cre in deletion of certain target genes.

Although more severe dysplasia was observed in the Ad-Cre/AAV-VEGF-injected brain of $A l k 1^{2 f / 2 f}$ mice than $E n g^{2 f / 2 f}$ mice, there were more dysplastic vessels formed per copy of Eng deletion than that of Alkl. This finding suggests that more severe dysplasia might develop in $E n g^{2 f / 2 f}$ mice if equivalent copies of Eng were deleted as compared to $A l k 1^{2 f / 2 f}$ mice. To improve deletion of the Eng gene, higher doses of Ad-Cre or other conditional Cre delivery approaches, such as an AAV-Cre vector and the ROSA-CreER (ER: estrogen receptor) system in which Cre expression is induced by tamoxifen treatment, may be utilized. AAV viral vector has been known to express exogenous protein longer than Ad viral vector following transduction [20]. In the ROSA-CreER system, Cre becomes ubiquitously and robustly active upon treatment of a chemical compound tamoxifen [21], and the Cre expression can also be enhanced by multiple treatments of tamoxifen. In addition, employing Eng ${ }^{2 f /}$ mice through cross-breeding between $E n g^{2 f / 2 f}$ and $E_{n g^{+/-}}$mice might be an option to achieve efficient homozygous deletion of Eng.

Evidence obtained from analysis of human AVM indicates that haploinsufficiency of ENG may not be sufficient to trigger lesion development. A case report of a HHT1 patient showed a 50\% reduction in the endoglin/ PECAM-1 ratio in the presumed normal blood vessels adjacent to lung and brain AVM [22]. In sporadic human bAVM tissue, there was no gross defect in endoglin expression in the endothelium [23]. We found that in mice, despite minimal ( $~ 1 \%)$ deletion of Eng in the brain of AdCre-treated $E n g^{2 f / 2 f}$ mice, more severe cerebrovascular dysplasia formed after VEGF stimulation compared to 
Eng ${ }^{+/-}$mice, i.e., $50 \%$ Eng deletion [5]. Eng-null ECs were found in the brain of Ad-Cre-treated Eng ${ }^{2 f / 2 f}$ mice, suggesting homozygous deletion of Eng in these cells causing mosaicism (fig. 3). This mosaic expression of ENG might be due to the accessibility of Eng to Cre that can be affected by transduction efficiency of Ad-Cre and effectiveness of Cre in mediating recombination of the loxP sites in the floxed allele. We have demonstrated in our previous study [8] that injection of Ad-Cre $\left(2 \times 10^{7} \mathrm{PFU}\right)$ into the basal ganglia of R26R mice resulted in targeted gene deletion in $51 \%$ of ECs. Further, the efficiency of Cremediated gene deletion varies among genes and is dependent on the genomic position of the loxP-containing targeted allele [24]. We found that Cre is relatively ineffective in mediating recombination of the loxP sites in the Engfloxed allele. Therefore, the homozygous deletion of Eng might occur in a small percentage of ECs. Thus, more dysplasia in the brain of Ad-Cre-treated Eng ${ }^{2 f / 2 f}$ mice suggests that homozygous mutation of Eng in a small number of ECs is sufficient to cause cerebrovascular malformations upon angiogenic stimulation. In addition, homozygous mutations appear to be an operant mechanism in the development of cavernous malformation, a related cerebrovascular disease causing hemorrhagic stroke $[25$, 26]. Additional study is thus needed to address mosaic gene expression patterns in human bAVM.
In summary, the comparison of heterozygous and homozygous Eng deletion in our mouse model of vascular malformation demonstrates that there is a distinct difference in lesional phenotype induction between heterozygous and homozygous gene deletion. While the $\mathrm{Eng}^{+/-}$ mice presented with only minimal irregular capillaries, the Ad-Cre-treated $E n g^{2 f / 2 f}$ mice with homozygous Eng deletion in a few ECs developed macroscopic level of dysplasia after VEGF stimulation. Our data are consistent with the hypothesis that the disease is contingent on loss of both gene copies; further study is needed to examine whether loss of heterozygosity (homozygous gene deletion) in ECs exists in human bAVM.

\section{Acknowledgements}

The authors thank Tony Pourmohamad for assistance with statistical analysis, Douglas A. Marchuk (Duke University Medical Center) for critical discussion and review of the manuscript, Voltaire Gungab for assistance with manuscript preparation, and the other members of the UCSF BAVM Study Project (http://avm. ucsf.edu) for their support. This study was supported in part by grants from the National Institutes of Health, T32 GM008440 (E.W.), R01 NS027713 (W.L.Y.), R21 NS070153 (H.S.), and P01NS044155 (W.L.Y. and H.S.); from the American Heart Association, AHA SDG 0535018N (H.S.); and from the British Heart Foundation (H.M.A.).

\section{References}

1 Mikhak B, Weinsheimer S, Pawlikowska L, Poon A, Kwok PY, Lawton MT, Chen Y, Zaroff JG, Sidney S, McCulloch CE, Young WL, Kim H: Angiopoietin-like 4 (ANGPTL4) gene polymorphisms and risk of brain arteriovenous malformations. Cerebrovasc Dis 2011;31:338-345.

2 Shovlin CL: Hereditary haemorrhagic telangiectasia: pathophysiology, diagnosis and treatment. Blood Rev 2010;24:203-219.

-3 Letteboer TG, Mager JJ, Snijder RJ, Koeleman BP, Lindhout D, Ploos van Amstel JK, Westermann CJ: Genotype-phenotype relationship in hereditary haemorrhagic telangiectasia. J Med Genet 2006;43:371-377.

4 Lebrin F, Deckers M, Bertolino P, Ten Dijke P: TGF-beta receptor function in the endothelium. Cardiovasc Res 2005;65:599-608.

5 Hao Q, Zhu Y, Su H, Shen F, Yang GY, Kim $\mathrm{H}$, Young WL: VEGF induces more severe cerebrovascular dysplasia in Endoglin ${ }^{+/}$ than in $\mathrm{Alk1}^{+/-}$mice. Transl Stroke Res 2010; 1:197-201.

-6 Attia W, Tada T, Hongo K, Nagashima H, Takemae T, Tanaka Y, Kobayashi S: Microvascular pathological features of immediate perinidal parenchyma in cerebral arteriovenous malformations: giant bed capillaries. J Neurosurg 2003;98:823-827.

7 Sato S, Kodama N, Sasaki T, Matsumoto M, Ishikawa T: Perinidal dilated capillary networks in cerebral arteriovenous malformations. Neurosurgery 2004;54:163-168; discussion 168-170.

-8 Walker EJ, Su H, Shen F, Choi EJ, Oh SP, Chen G, Lawton MT, Kim H, Chen Y, Chen W, Young WL: Arteriovenous malformation in the adult mouse brain resembling the human disease. Ann Neurol 2011;69:954-962.

-9 Park SO, Lee YJ, Seki T, Hong KH, Fliess N, Jiang Z, Park A, Wu X, Kaartinen V, Roman BL, Oh SP: ALK5- and TGFBR2-independent role of ALK1 in the pathogenesis of hereditary hemorrhagic telangiectasia type 2 (HHT2). Blood 2008;111:633-642.

10 Allinson KR, Carvalho RL, van den Brink S, Mummery CL, Arthur HM: Generation of a floxed allele of the mouse Endoglin gene. Genesis 2007;45:391-395.

1 Su H, Lu R, Kan YW: Adeno-associated viral vector-mediated vascular endothelial growth factor gene transfer induces neovas- cular formation in ischemic heart. Proc Nat Acad Sci USA 2000;97:13801-13806.

12 Shen F, Su H, Liu W, Kan YW, Young WL, Yang GY: Recombinant adeno-associated viral vector encoding human VEGF165 induces neomicrovessel formation in the adult mouse brain. Front Biosci 2006;11:31903198.

13 Walker EJ, Shen F, Young WL, Su H: Cerebrovascular casting of adult mouse for 3D imaging and morphological analysis. J Vis Exp 2011;57:2958.

- 14 Hao Q, Su H, Marchuk DA, Rola R, Wang Y, Liu W, Young WL, Yang GY: Increased tissue perfusion promotes capillary dysplasia in the ALK1-deficient mouse brain following VEGF stimulation. Am J Physiol Heart Circ Physiol 2008;295:H2250-H2256.

15 Zhang ZG, Zhang L, Jiang Q, Zhang R, Davies $\mathrm{K}$, Powers C, Bruggen N, Chopp M: VEGF enhances angiogenesis and promotes blood-brain barrier leakage in the ischemic brain. J Clin Invest 2000;106:829-838.

16 Soriano P: Generalized lacZ expression with the ROSA26 Cre reporter strain. Nat Genet 1999;21:70-71. 
17 Zhou L, Nepote V, Rowley DL, Levacher B, Zvara A, Santha M, Mi QS, Simonneau M, Donovan DM: Murine peripherin gene sequences direct Cre recombinase expression to peripheral neurons in transgenic mice. FEBS Lett 2002;523:68-72.

- 18 Licht AH, Raab S, Hofmann U, Breier G: Endothelium-specific Cre recombinase activity in flk-1-Cre transgenic mice. Dev Dyn 2004; 229:312-318.

19 Wang P, Wang SM, Hsieh CJ, Chien CL: Neural expression of alpha-internexin promoter in vitro and in vivo. J Cell Biochem 2006;97: 275-287.

20 Chu D, Sullivan CC, Weitzman MD, Du L, Wolf PL, Jamieson SW, Thistlethwaite PA: Direct comparison of efficiency and stability of gene transfer into the mammalian heart using adeno-associated virus versus adenovirus vectors. J Thorac Cardiovasc Surg 2003;126:671-679.
21 Park SO, Wankhede M, Lee YJ, Choi EJ, Fliess N, Choe SW, Oh SH, Walter G, Raizada MK, Sorg BS, Oh SP: Real-time imaging of de novo arteriovenous malformation in a mouse model of hereditary hemorrhagic telangiectasia. J Clin Invest 2009;119:34873496.

22 Bourdeau A, Cymerman U, Paquet ME, Meschino W, McKinnon WC, Guttmacher AE, Becker L, Letarte M: Endoglin expression is reduced in normal vessels but still detectable in arteriovenous malformations of patients with hereditary hemorrhagic telangiectasia type 1. Am J Pathol 2000;156:911-923.

3 Matsubara S, Bourdeau A, terBrugge KG, Wallace C, Letarte M: Analysis of endoglin expression in normal brain tissue and in cerebral arteriovenous malformations. Stroke 2000;31:2653-2660.
24 Vooijs M, Jonkers J, Berns A: A highly efficient ligand-regulated Cre recombinase mouse line shows that LoxP recombination is position dependent. EMBO Rep 2001;2: 292-297.

25 Chan AC, Drakos SG, Ruiz OE, Smith AC, Gibson CC, Ling J, Passi SF, Stratman AN, Sacharidou A, Revelo MP, Grossmann AH, Diakos NA, Davis GE, Metzstein MM, Whitehead KJ, Li DY: Mutations in 2 distinct genetic pathways result in cerebral cavernous malformations in mice. J Clin Invest 2011;121:1871-1881.

26 Akers AL, Johnson E, Steinberg GK, Zabramski JM, Marchuk DA: Biallelic somatic and germline mutations in cerebral cavernous malformations (CCM): evidence for a two-hit mechanism of CCM pathogenesis. Hum Mol Genet 2009;18:919-930. 